\title{
Bone management in Japanese patients with prostate cancer: hormonal therapy leads to an increase in the FRAX score
}

\author{
Takashi Kawahara ${ }^{1,2+}$, Shusei Fusayasu ${ }^{1 \dagger}$, Koji Izumi', Yumiko Yokomizo', Hiroki Ito ${ }^{1}$, Yusuke Ito ${ }^{1}$, Kayo Kurita', \\ Kazuhiro Furuya ${ }^{1}$, Hisashi Hasumi ${ }^{1}$, Narihiko Hayashi ${ }^{1}$, Yasuhide Myoshi $^{2}$, Hiroshi Miyamoto ${ }^{3}$, Masahiro Yao ${ }^{1}$ \\ and Hiroji Uemura ${ }^{1,2^{*}}$
}

\begin{abstract}
Background: Osteoporosis is a common consequence of androgen deprivation therapy (ADT) for prostate cancer. Up to $20 \%$ of men on ADT have suffered from fractures within 5 years. The WHO Fracture Risk Assessment Tool (FRAX) has been utilized to predict the 10-year probability of major osteoporotic and hip fracture. However, to date, no large studies assessing the utility of the FRAX score in prostate cancer patients with or without ADT have been performed. We herein evaluated the impact of ADT on the FRAX score in prostate cancer patients.

Methods: The assessment of the FRAX score was performed in a total of 1220 prostate cancer patients, including patients who underwent brachytherapy $(n=547)$, radical prostatectomy $(n=200)$, external beam radiation therapy $(n=264)$ and hormonal therapy alone $(n=187)$ at Yokohama City University Hospital (Yokohama, Japan). We evaluated the effect of ADT on the FRAX score.

Results: Using the FRAX model, the median and mean 10-year probability of a major osteoporotic fracture according to the clinical risk factors alone was $7.9 \%(8.8 \pm 4.3 \%)$, while the 10-year probability of hip fracture risk was $2.7 \%(3.5 \pm 3.1 \%)$. In the ADT group, the duration of ADT was correlated with both major osteoporotic risk and hip fracture risk $\left(R^{2}=0.141, p<0.001\right.$ and $R^{2}=0.166, p<0.001$, respectively). A comparison between the ADT $(n=187)$ and non-ADT $(n=399)$ groups demonstrated that the major fracture risk was $>20 \%$ higher and the hip fracture risk was $>3 \%$ higher in the ADT group than in the non-ADT group (ADT: 10 (5.3 \%) and 118 (63.1\%), non-ADT 13 (3.3\%) and 189 (47.4\%), $p<0.001$ and $p<0.001$, respectively).
\end{abstract}

Conclusions: These results suggested that the longer duration of ADT led to an increased FRAX score, and the FRAX score may be a predictor of bone management treatment, particularly in prostate cancer patients.

Keywords: Androgen deprivation therapy, FRAX, Prostate cancer, Bone fracture

\section{Background}

Following the widespread implementation of PSA screening, the incidence of prostate cancer has been increasing in Japan [1,2]. By the end of 2015, 98,400 men will have been newly diagnosed and 12,200 men will

\footnotetext{
* Correspondence: hu0428@yokohama-cu.ac.jp

${ }^{\dagger}$ Equal contributors

'Department of Urology, Yokohama City University, Graduate School of Medicine, Yokohama, Japan

${ }^{2}$ Department of Urology and Renal Transplantation, Yokohama City University Medical Center, 4-57 Urafune-cho, Minami-ku, Yokohama, Kanagawa 232-0024, Japan

Full list of author information is available at the end of the article
}

have died of prostate cancer. Many patients diagnosed with prostate cancer are elderly and must be treated with hormonal therapy [3]. Men with prostate cancer are often at risk for other age-related adverse events, such as hip fractures. The risk of hip fractures can increase in men with prostate cancer because the bone density often decreases due to androgen deprivation therapy (ADT), occult bone metastases, or a combination thereof [4-8]. The mechanism is believed to be due to a decrease in sexual hormone levels, which induces receptor activator of nuclear factor-kappa-B ligand (RANKL) expression from osteoblasts. Consequently, 
osteoclasts are involved in bone resorption [9]. The skeleton is the third most common site of metastatic cancers, and one-third to one-half of all cancers metastasize to the bone. In prostate cancer, bone metastasis is the most common site for tumor development [10]. Osteoporosis or low bone mineral density (BMD) is a highly prevalent health problem in the elderly as well as in prostate cancer patients treated with ADT. The FRAX score is a fracture risk assessment tool developed by the World Health Organization (WHO) to predict the fracture risk of patients according to clinical risk factors alone or in combination with BMD at the femoral neck [11]. It is a computer-based algorithm which provides the 10-year probability of hip and major osteoporotic fractures (e.g., clinical spine, forearm, hip, or shoulder fracture) according to age, sex, body mass index, and clinical risk factors $[12,13]$. There have been no proven methods for predicting pathologic fractures in patients with skeletal metastasis thus far [14].

The fracture risk varies depending on the geographic location and ethnicity, and the FRAX algorithm has been calibrated to account for this [11]. Algorithms are available for diverse ethnic groups, including Asians, based largely on data from Japan and China [13]. Despite its importance, there have been few studies which investigated the fracture risk among Asians with prostate cancer living in Japan [13]. The present study evaluates the risk of developing hip fractures in Japanese men treated at one institute for prostate cancer [4] by examining whether or not ADT could influence the FRAX score.

\section{Methods}

\section{Patients}

A total of 1220 patients were enrolled in this study. All patients were pathologically diagnosed with prostate cancer and followed-up at Yokohama City University Hospital (Yokohama, Japan) and the FRAX score was calculated once during prostate cancer therapy. Written informed consent was obtained from all patients and this study protocol was approved by the Institutional Review Board at Yokohama City University Hospital.

The study cohort comprised 547 (44.8\%) patients who received brachytherapy, 200 (16.4\%) who received radical prostatectomy, 267 (21.9\%) who received external beam radiation therapy (EBRT), 187 (15.3\%) who received ADT monotherapy, and 19 (1.6\%) who received active surveillance. Excluding the ADT monotherapy group, 615 $(50.4 \%)$ patients received ADT in combination with surgical or radiation therapy. (Table 1) In the present study, EBRT was performed as a high-dose intensity-modulated radiation therapy. Some of the patients in the hormonal therapy group had bone metastasis at the time of diagnosis. To exclude bias, men with castration-resistant prostate cancer were not included in the present study. Data from
Table 1 Patients' background

\begin{tabular}{ll}
\hline Variables & number (\%) \\
\hline Total & $1220(100.0 \%)$ \\
Brachy Therapy & $547(44.8 \%)$ \\
with neo-adjuvant HTx & $336(27.5 \%)$ \\
without HTx & $211(17.3 \%)$ \\
Total Prostatectomy & $200(16.4 \%)$ \\
with adjuvant HTx & $50(4.1 \%)$ \\
without HTx & $150(12.3 \%)$ \\
EBRT & $267(21.9 \%)$ \\
with HTx & $229(18.8 \%)$ \\
without HTx & $38(3.1 \%)$ \\
Hormonal Tx only & $187(15.3 \%)$ \\
Active surveillance & $19(1.6 \%)$ \\
\hline
\end{tabular}

each subject were entered into the FRAX algorithm and 10-year fracture probabilities were calculated using the clinical risk factors (CRFs) alone. Due to the differences between the FRAX score alone or in combination with $\mathrm{BMD}$, we excluded the FRAX score using the BMD measurement. BMD was measured using dual-energy X-ray absorptiometry of the femoral neck.

The FRAX tool (available at http://www.shef.ac.uk/ FRAX/tool.jsp?lang=en) was used to compute the probability of a major osteoporotic event and hip fracture. The date of birth, weight $(\mathrm{kg})$, height $(\mathrm{cm})$, and a yes or no response for various CRFs were entered into the FRAX questionnaire, and the fracture probability was calculated for each subject. Childhood fractures reported by the subjects were not considered to be fragility fractures.

\section{Statistical analysis}

All continuous variables were expressed as the means \pm SD. The numerical data were compared using Student's $t$-test. The correlation between 148 variables was determined using Spearman's correlation coefficient. A $p$-value 149 of 0.05 or less was considered to be statistically significant.

\section{Results}

A total of 1220 men were recruited to participate in this study. Their demographic characteristics are presented in Table 2. There were no differences in terms of age, body weight, body height, previous fractures, parent fractured hip, current smoking, use of glucocorticoids, rheumatoid arthritis, secondary osteoporosis, and alcohol consumption of 3 or more units per day, which are risk factors for fracture that are included in the FRAX score (Table 2).

Using the FRAX model, the median and mean 10-year probability of a major osteoporotic fracture according to the CRFs alone was $7.9 \%(8.8 \pm 4.3 \%)$, while the 10-year probability of hip fracture risk was $2.7 \%(3.5 \pm 3.1 \%)$. 
Table 2 Results in each therapy with prostate cancer patients

\begin{tabular}{llllll}
\hline Number (\%) or median (Mean \pm SD) & Total (1220) & Brachy Tx (547) & Prostatectomy (200) & EBRT (267) & Hormonal monotherapy (187) \\
\hline Variables & $74(73.3 \pm 7.1)$ & $73(72.1 \pm 6.4)$ & $72.5(72.1 \pm 7.1)$ & $75(74.6 \pm 6.5)$ & $77(76.3 \pm 8.2)$ \\
\hline Age & $64(64.3 \pm 8.9)$ & $64(64.6 \pm 8.7)$ & $63.6(63.7 \pm 8.3)$ & $65(65.1 \pm 9.2)$ & $63(63.3 \pm 9.9)$ \\
Weight (kg) & $165(165.5 \pm 6.0)$ & $165(165.7 \pm 6.1)$ & $165(165.4 \pm 5.8)$ & $166(165.7 \pm 5.9)$ & $165(164.6 \pm 6.0)$ \\
Height (cm) & $255(20.9 \%)$ & $111(20.2 \%)$ & $40(20.0 \%)$ & $63(23.6 \%)$ & $35(18.7 \%)$ \\
Previous fracture & $90(7.4 \%)$ & $38(6.9 \%)$ & $19(8.5 \%)$ & $18(6.7 \%)$ & $12(6.4 \%)$ \\
Parent Fractured Hip & $138(11.3 \%)$ & $71(13.0 \%)$ & $26(13.0 \%)$ & $22(8.2 \%)$ & $16(8.6 \%)$ \\
Current Smoking & $38(3.1 \%)$ & $3(0.5 \%)$ & $4(2.0 \%)$ & $8(3.0 \%)$ & $19(10.2 \%)$ \\
Glucocorticoid & $13(1.1 \%)$ & $3(0.5 \%)$ & $3(1.5 \%)$ & $3(1.1 \%)$ & $4(2.1 \%)$ \\
Rheumatoid arthritis & $108(8.9 \%)$ & $45(8.2 \%)$ & $20(10.0 \%)$ & $26(9.7 \%)$ & $18(9.6 \%)$ \\
Secondary osteoporosis & $380(31.1 \%)$ & $174(31.8 \%)$ & $72(36.0 \%)$ & $95(35.6 \%)$ & $38(20.3 \%)$ \\
Alcohol 3 or more units per day $\%)$
\end{tabular}

Forty-nine patients $(4.0 \%)$ had a major osteoporotic fracture risk of more than $20 \%$ and 643 patients $(52.7 \%$ ) had a hip fracture risk of more than $3 \%$. A comparison between the ADT monotherapy $(n=187)$ and non-ADT $(n=399)$ groups showed that the major osteoporotic fracture risk > $20 \%$ and hip fracture risk $>3 \%$ was significantly higher in the ADT group than in the non-ADT group (ADT: 10 (5.3\%) and 118 (63.1\%), non-ADT 13 (3.3\%) and 189 (47.4\%), $p<0.001$ and $p<0.001$, respectively).

The major osteoporotic risk of patients who received periodical ADT in combination with brachytherapy tended to be higher than that of the patients who did not receive $\operatorname{ADT}(p=0.12)$. On the other hand, the hip fracture risk of the patients who received ADT in combination with brachytherapy was significantly higher than that of the patients who did not receive ADT $(p=0.04)$ (Fig. 1).

Regarding the therapies for prostate cancer, the major osteoporotic risk was $7.9 \%(8.8 \pm 4.3 \%)$ in the brachytherapy group, $8.2 \%(9.3 \pm 5.2 \%)$ in the radical prostatectomy group, $9.2 \%(19.2 \pm 5.0)$ in the EBRT group, and $9.1 \%(10.4 \pm 5.4 \%)$ in the ADT group. The EBRT and ADT monotherapy groups showed significantly higher major osteoporotic risk than the brachytherapy group $(p<0.001$ and $p<0.001$, respectively). The hip fracture risk was $2.7 \%(3.5 \pm 3.1 \%)$ in the brachytherapy group, $2.9 \%(3.7 \pm 3.3 \%)$ in the radical prostatectomy group, $3.6 \%(4.5 \pm 4.0 \%)$ in the EBRT group, and $4.0 \%(5.2 \pm$ $4.6 \%)$ in the ADT group. The EBRT and ADT groups showed significantly higher hip fracture risk than both the brachytherapy and prostatectomy groups $(p<0.001$, $p<0.001$ and $p=0.02, p<0.001$, respectively) (Fig. 2).

The major osteoporotic risk $>20 \%$ and hip fracture risk $>3 \%$ were 2.7 and $46.6 \%$ in the brachytherapy group, 3.5 and $48.5 \%$ in the radical prostatectomy group, 5.6 and $60.7 \%$ in the EBRT group, and 5.9 and $70.1 \%$ in the ADT group.
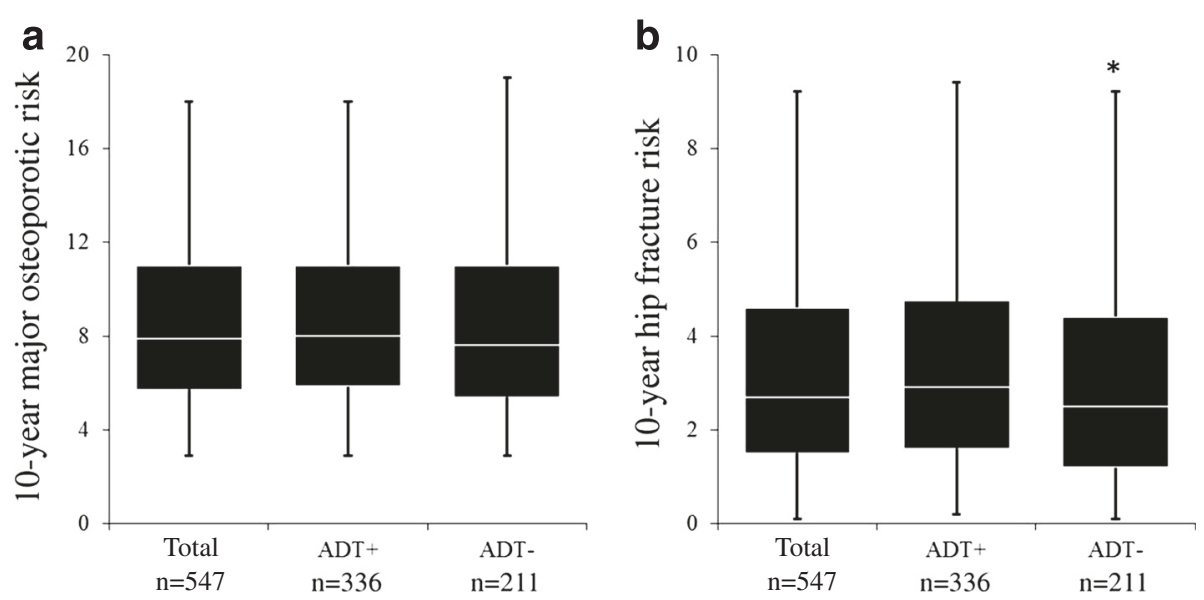

Fig. 1 The FRAX score with or without ADT in the patients who received brachytherapy. The FRAX score between prostate cancer patients who received brachytherapy with ADT and those without ADT was compared. a According to the 10-year major osteoporotic risk, the ADT group showed a tendency toward a higher FRAX score $(p=0.12)$. b According to the 10-year hip fracture risk, the ADT group showed a significantly higher FRAX score than the non-ADT group $(p=0.04)$ 

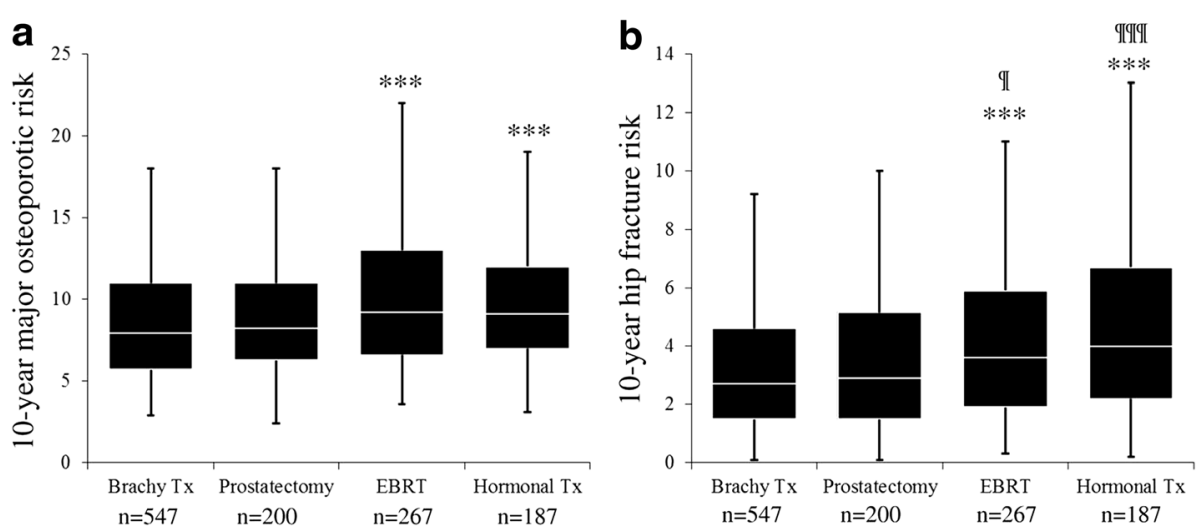

Fig. 2 The FRAX score among various prostate cancer therapies. a: The 10-year major osteoporotic risk. The EBRT and ADT monotherapy groups showed significantly higher FRAX scores than the brachytherapy group $(p<0.001, p<0.001$, respectively). b: The 10-year hip fracture risk. The EBRT and ADT monotherapy groups showed higher FRAX scores than both the brachytherapy group $(p<0.001, p<0.001$, respectively) and the prostatectomy group $(p<0.05, p<0.001$, respectively)

When analyzed in the ADT monotherapy group, the duration of ADT correlated with both the major osteoporotic risk and hip fracture risk $\left(\mathrm{R}^{2}=0.141, p<0.001\right.$ and $\mathrm{R}^{2}=0.166, p<0.001$, respectively) (Fig. 3 ).

\section{Discussion}

This study revealed that ADT increased the FRAX score and that the duration of ADT correlated with the FRAX score. In particular for prostate cancer patients treated with hormonal therapy, fractures are a major complication which can influence both the activities of daily life and life expectancy. Although the median 10-year probability of major osteoporotic and hip fracture risks were not higher than expected, 7.9 and $2.7 \%$, respectively, ADT elevated those probabilities as expected. It is plausible that patients who received $\mathrm{ADT}$ were comparatively older than those who received brachytherapy or RRP. We found that the
FRAX score correlated with the duration of ADT. In most of the patients in the brachytherapy-treated group, ADT was performed within one year. Consequently, there were no large differences occurred within the brachytherapy group regardless of whether the patients received ADT or not. ADT, a common treatment option for patients with prostate cancer that reduces circulating testosterone levels, has a detrimental effect on BMD, leading to a substantial increase in the fracture risk $[8,15,16]$. Men undergoing ADT are four times more likely to develop significant bone deficiency $[8,15]$. ADT is known to cause a decrease in $\mathrm{BMD}$, and therefore patients who receive ADT should be assumed to have secondary osteoporosis when calculating the fracture risk using the FRAX score $[2,11]$. It has been well established that fractures are associated with significant morbidity and mortality. This study showed that the increase in FRAX score by ADT correlated with the duration
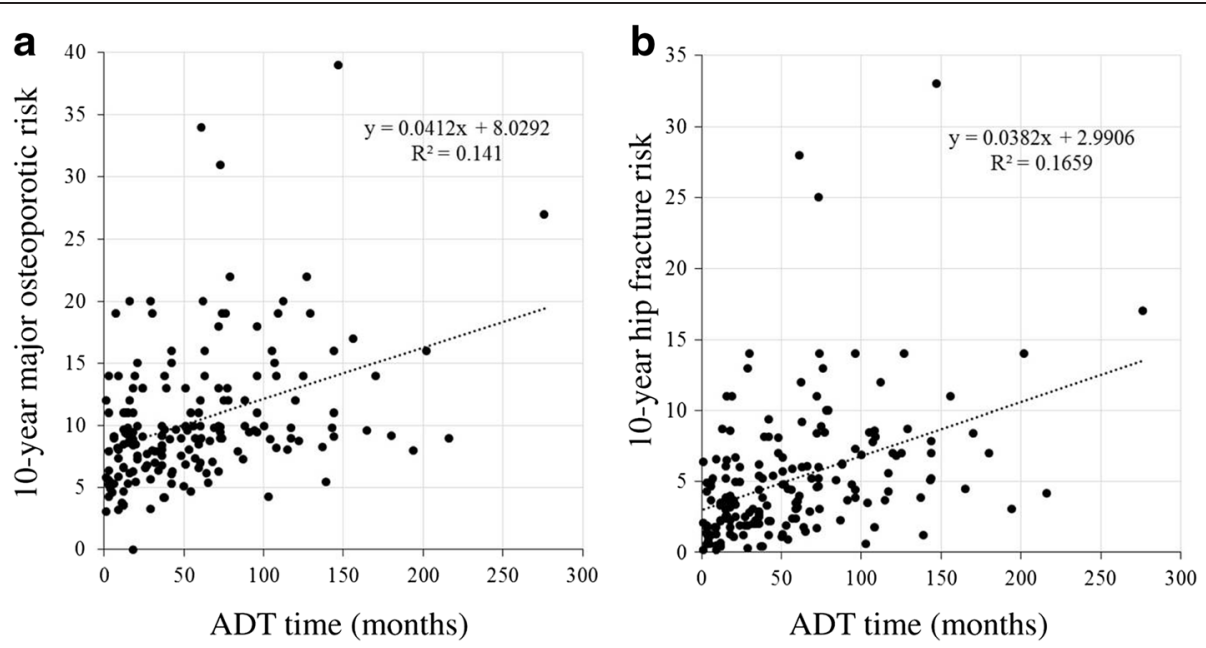

Fig. 3 The FRAX score correlated with the duration of ADT. a: The 10-year major osteoporotic risk was positively correlated with the duration of ADT $\left(R^{2}=0.141, p<0.0001\right)$. b: The 10-year hip fracture risk was positively correlated with the duration of $\operatorname{ADT}\left(R^{2}=0.1659, p<0.0001\right)$ 
of ADT. During ADT, it might be recommended that patients, especially those who have received ADT for longer periods of time, perform regular muscle-strengthening exercises or increase their vitamin D intake [17].

Although ADT use was positively correlated with the FRAX score $\left(R^{2}=0.141\right)$, the correlation was not so high. We speculate that the relatively weak correlation resulted from the use of the FRAX score for assessment rather than the DEXA score. We are presently conducting another study to reveal the correlation between the ADT time and the risk of fracture using the DEXA score and some bone related biomarkers. Although there were some indications for intervention to prevent fracture, we set the cut-off point as $20 \%$ in patients with major osteoporosis and $3 \%$ in patients with hip fracture because a 10-year probability of major osteoporotic risk $>20 \%$ and hip fracture $>3 \%$ is considered to represent a clinically-relevant degree of risk [11] according to the American National Osteoporosis Foundation Clinician's Guide to Prevention and Treatment of Osteoporosis. Our study also found that the ADT group showed a significantly higher fracture risk, which requires fracture prevention.

There are two limitations associated with the present study. First, we assessed the FRAX score and did not determine the patients' real fracture incidences over the 10-year follow-up period. The FRAX was developed by the WHO to predict the fracture risk; however, this score has some ethnicity-related differences, although there are algorithms available for various ethnic groups. In addition, this score is typically used during the clinical courses. Therefore, assessing the fracture risk using the FRAX score is valuable. Second, we did not include the BMD score in this study. BMD is a useful tool to predict fractures and initiate the intervention for bone management $[12,13]$. The National Osteoporosis Foundation and other groups have recommended guidelines for BMD testing according to clinical factors. Additionally, the FRAX score may be used with or without BMD. In the present study we did not use BMD testing in our assessment of the FRAX score. In several cases, we also performed dual-energy X-ray absorptiometry (DEXA) to calculate the BMD and found that the FRAX score was reduced when we used BMD (data not shown). In the present study FRAX was therefore used as a screening tool to determine whether or not BMD testing should be performed. The 10-year probability of a major osteoporotic fracture increases when the femur neck Tscores are added to the CRFs in the FRAX algorithm, and this population has a high fracture probability even in the absence of CRFs [18, 19]. However, in clinical use, not all patients undergo DEXA due to the medical costs. Accordingly, we recommend the use of the FRAX score for prostate cancer patients, especially those treated with ADT, as a screening tool in addition to bone management therapy. The FRAX algorithm without the BMD values was superior to BMD alone in identifying the patients at a higher risk of fractures. Therefore, patients with high FRAX scores should receive DEXA in order to avoid additional bone management therapy.

\section{Conclusion}

The duration of ADT can influence the FRAX score and the ADT group showed a significantly higher FRAX score and an increased need for additional bone management treatment. This large study is needed to show that ADT influenced the FRAX scores.

\section{Abbreviations \\ ADT, Androgen deprivation therapy; FRAX, fracture risk assessment tool; $B M D$, bone mineral density; DEXA, dual-energy $X$-ray absorptiometry; CRF, clinical risk factors; RRP, radical retropubic prostatectomy}

\section{Acknowledgement \\ We thank S Honjo, T lijima and S Uchiyama for statistical survey. This study was supported by a MEXT/JSPS KAKENHI Grant.}

\section{Availability of data and materials}

Due to ethical restrictions, the raw data underlying this paper that was used in this study is available upon request to from the corresponding author.

\section{Authors' contributions}

TK and HU contributed to drafting the manuscript, HU and SF were involved in the design of the study. $\mathrm{KI}, \mathrm{YY}, \mathrm{HI}, \mathrm{Yl}, \mathrm{KK}, \mathrm{KF}, \mathrm{HH}, \mathrm{NH}, \mathrm{YM}$, and $\mathrm{HM}$ were study investigators, MY was responsible for clinical trial management. TK was responsible for the statistical analysis. All authors read and approved the final manuscript.

\section{Competing interests}

The authors declare that they have no competing interests.

\section{Author details}

${ }^{1}$ Department of Urology, Yokohama City University, Graduate School of Medicine, Yokohama, Japan. ²Department of Urology and Renal Transplantation, Yokohama City University Medical Center, 4-57 Urafune-cho, Minami-ku, Yokohama, Kanagawa 232-0024, Japan. ${ }^{3}$ Departments of Pathology and Urology, Johns Hopkins University School of Medicine, Baltimore, USA.

Received: 9 May 2015 Accepted: 9 May 2016

Published online: 17 June 2016

\section{References}

1. Polascik TJ, Oesterling JE, Partin AW. Prostate specific antigen: a decade of discovery-what we have learned and where we are going. J Urol. 1999; 162(2):293-306.

2. Kawahara T, Ishiguro H, Hoshino K, Teranishi J, Miyoshi Y, Kubota Y, Uemura $\mathrm{H}$. Analysis of NSAID-activated gene 1 expression in prostate cancer. Urol Int. 2010;84(2):198-202.

3. Rosenberg L, Lawlor GO, Zenlea T, Goldsmith JD, Gifford A, Falchuk KR, Wolf $J$, Cheifetz AS, Robson SC, Moss AC. Predictors of endoscopic inflammation in patients with ulcerative colitis in clinical remission. Inflamm Bowel Dis. 2013:19(4):779-84

4. Valery R, Mendenhall NP, Nichols Jr RC, Henderson R, Morris CG, Su Z, Mendenhall WM, Williams CR, Li Z, Hoppe BS. Hip fractures and pain following proton therapy for management of prostate cancer. Acta Oncol. 2013;52(3):486-91.

5. Wadhwa VK, Weston R, Mistry R, Parr NJ. Long-term changes in bone mineral density and predicted fracture risk in patients receiving androgen-deprivation therapy for prostate cancer, with stratification of treatment based on presenting values. BJU Int. 2009;104(6):800-5. 
6. Hatano T, Oishi Y, Furuta A, Iwamuro S, Tashiro K. Incidence of bone fracture in patients receiving luteinizing hormone-releasing hormone agonists for prostate cancer. BJU Int. 2000;86(4):449-52.

7. Melton 3rd LJ, Lieber MM, Atkinson EJ, Achenbach SJ, Zincke H, Therneau TM, Khosla S. Fracture risk in men with prostate cancer: a population-based study. J Bone Mineral Res Off J Am Soc Bone Mineral Res. 2011;26(8):1808-15.

8. Shahinian VB, Kuo YF, Freeman JL, Goodwin JS. Risk of fracture after androgen deprivation for prostate cancer. N Engl J Med. 2005;352(2):154-64.

9. Schulman C, Irani J, Aapro M. Improving the management of patients with prostate cancer receiving long-term androgen deprivation therapy. BJU Int. 2012;109 Suppl 6:13-21.

10. Michaeli DA, Inoue K, Hayes WC, Hipp JA. Density predicts the activitydependent failure load of proximal femora with defects. Skelet Radiol. 1999; 28(2):90-5.

11. Kanis JA, Oden A, Johansson H, Borgstrom F, Strom O, McCloskey E. FRAX and its applications to clinical practice. Bone. 2009;44(5):734-43.

12. Johansson H, Kanis JA, Oden A, Johnell O, McCloskey E. BMD, clinical risk factors and their combination for hip fracture prevention. Osteoporosis Int J Established Result Cooperation Between Eur Foundation Osteoporosis National Osteoporosis Foundation USA. 2009;20(10):1675-82.

13. Kuruvilla K, Kenny AM, Raisz LG, Kerstetter JE, Feinn RS, Rajan TV. Importance of bone mineral density measurements in evaluating fragility bone fracture risk in Asian Indian men. Osteoporosis Int J Estab Result Cooperation Between Eur Foundation Osteoporosis National Osteoporosis Foundation USA. 2011;22(1):217-21.

14. Hipp JA, Springfield DS, Hayes WC. Predicting pathologic fracture risk in the management of metastatic bone defects. Clin Orthop Relat Res. 1995;312: $120-35$.

15. James 3rd H, Aleksic I, Bienz MN, Pieczonka C, lannotta P, Albala D, Mariados N, Mouraviev V, Saad F. Comparison of fracture risk assessment tool score to bone mineral density for estimating fracture risk in patients with advanced prostate cancer on androgen deprivation therapy. Urology. 2014;84(1):164-8.

16. Saad F, Abrahamsson PA, Miller K. Preserving bone health in patients with hormone-sensitive prostate cancer: the role of bisphosphonates. BJU Int. 2009:104(11):1573-9.

17. Cosman F, de Beur SJ, LeBoff MS, Lewiecki EM, Tanner B, Randall S, Lindsay R, National Osteoporosis F. Clinician's Guide to Prevention and Treatment of Osteoporosis. Osteoporos Int. 2014;25(10):2359-81.

18. Lewiecki EM, Watts NB, McClung MR, Petak SM, Bachrach LK, Shepherd JA, Downs RW, Jr., International Society for Clinical D: Official positions of the international society for clinical densitometry. J Clin Endocrinol Metab. 2004; 89(8):3651-5.

19. Force USPST. Screening for osteoporosis in postmenopausal women: recommendations and rationale. Ann Intern Med. 2002;137(6):526-5.

\section{Submit your next manuscript to BioMed Central and we will help you at every step:}

- We accept pre-submission inquiries

- Our selector tool helps you to find the most relevant journal

- We provide round the clock customer support

- Convenient online submission

- Thorough peer review

- Inclusion in PubMed and all major indexing services

- Maximum visibility for your research

Submit your manuscript at www.biomedcentral.com/submit

C Biomed Central 